\title{
Nonlinear Dynamics of Controlled Synchronizations of Manipulator System
}

\author{
Qingkai Han, ${ }^{1,2}$ Hao Zhang, ${ }^{1}$ and Jinguo Liu ${ }^{2}$ \\ ${ }^{1}$ Dalian University of Technology, Dalian 116024, China \\ ${ }^{2}$ State Key Laboratory of Robotics, Shenyang Institute of Automation, Chinese Academy of Sciences, Shenyang 110016, China \\ Correspondence should be addressed to Qingkai Han; hanqingkai@dlut.edu.cn
}

Received 16 October 2013; Revised 20 November 2013; Accepted 21 November 2013; Published 29 January 2014

Academic Editor: Rongni Yang

Copyright (c) 2014 Qingkai Han et al. This is an open access article distributed under the Creative Commons Attribution License, which permits unrestricted use, distribution, and reproduction in any medium, provided the original work is properly cited.

\begin{abstract}
The nonlinear dynamics of the manipulator system which is controlled to achieve the synchronization motions is investigated in the paper. Firstly, the control strategies and modeling approaches of the manipulator system are given, in which the synchronization goal is defined by both synchronization errors and its derivatives. The synchronization controllers applied on the manipulator system include neuron synchronization controller, improved OPCL synchronization controller, and MRAC-PD synchronization controller. Then, an improved adaptive synchronized control strategy is proposed in order to estimate online the unknown structure parameters and state variables of the manipulator system and to realize the needed synchronous compensation. Furthermore, a robust adaptive synchronization controller is also researched to guarantee the dynamic stability of the system. Finally, the stability of motion synchronizations of the manipulator system possessing nonlinear component is discussed, together with the effect of control parameters and joint friction and others. Some typical motions such as motion bifurcations and the loss of synchronization of it are obtained and illustrated as periodic, multiperiodic, and/or chaotic motion patterns.
\end{abstract}

\section{Introduction}

A manipulator system can be viewed as a highly nonlinear, strong coupling, time-varying, and multivariable dynamic one. In some particular sites, synchronization motions are needed for the manipulator system to achieve expected tasks. The synchronization control strategies applied to the manipulator system are important to be reasonablly designed. So, the nonlinear dynamics of the controlled manipulator system in synchronization should be discovered specifically. The main purpose of the nonlinear dynamic research on manipulator system under synchronization control is to design a synchronous controller which can guarantee the synchronous stable characteristic. The controller should meet the requirement of trajectory tracking control accuracy. One of the special advantages of synchronization control is that it can keep the specified kinematics relationship of manipulator system in an easy way.

Controlled synchronization of manipulator system is also valuable in the mechanical research and engineering applications. Different from the traditional synergic control (i.e., coordination control associated with robot task assignment) and the coordinated control (i.e., force and position compliance control of robot), the controlled synchronization focuses on inertial characteristics, motion stiffness, and the rigid-flexible coupling characteristics of the manipulator system. The most important is to understand the dynamic behavior of the controlled system in synchronization. The synchronous control also needs to explore the synchronous tragedy and its stability and robustness when the manipulator system is different; in particular some nonlinearity and/or rigid-flexible coupling effort are concerned. Both the trajectory tracking error and synchronization error of manipulator system would converge to zero when using a controlled synchronization method, which is also useful to optimize the transient process of robot motion trajectory. More complicated motion patterns can be realized when using synchronization control, no matter the same or different structure, rigid or flexible links or joints of a manipulator system. In practices, the synchronization control strategies are also adopted to maintain more regular motions of the multiple industrial robots (such as for assembling, spraying, 
transporting, welding, etc.) and improve their trajectory tracking accuracies.

Nowadays, nonlinear dynamics of controlled synchronization of manipulator system is one of the important interests in the fields of machinery dynamics and nonlinear sciences [1-6]. The theory of self-synchronization, namely, vibration synchronization, was thoroughly and systematically studied by many researchers including Blekhman [2] and Wen et al. [7]. Self-synchronizations are used widely in designing vibratory machines and bring people remarkable economic benefits. Study on controlled synchronization and generalized synchronization of mechanical systems was extended during the past decades $[7,8]$. Recently, the theory of controlled synchronization is used to improve the control ability and control accuracy for many complicated mechanical systems, such as multiaxis machining tools, multirobotic coordination, and trajectory tracking of robots. Some representative achievements include the following. Koren [9] proposed a controlled synchronization strategy of a multiaxis machining tool where the cross-coupling control is used to achieve the multiaxis tracking synchronization and the synchronization errors are used to define the coordination ability of the machine. The research group leading by RodriguezAngeles and Nijmeijer [10] and Nijmeijer [11, 12] carried out external synchronization and internal synchronous controls of multirobot systems by using feedback controls. They proposed two new adaptive synchronization control methods to achieve the P-R-R planar parallel manipulators with uncertain parameters to guarantee the required trajectory tracking accuracy. Until now, many control strategies were also explored to achieve synchronizations of mechanical systems [13-20], including the controlled synchronization of speed of electromechanical systems consisting of double motors or multimotor running in a constant velocity ratio [7].

In addition, controlled synchronization of manipulators is critical in theoretical and engineering fields of mechanical system. The involved theoretical and technological results enclose the synchronous mechanisms, synchronization control strategies, chaotic synchronization controls, and so on. Many improved controlled synchronization methods are developed these years $[21,22]$. With the deepening study of nonlinear behaviors in many control domains [23], the nonlinear behavior of the manipulator due to the strong coupling has attracted more and more interest. For the controlled synchronization of manipulator system, the authors achieved many tasks including the nonlinear dynamic modeling, control strategies and nonlinear behavior of the controlled synchronization of manipulator systems, to satisfy the dynamic design and vibration suppression of manipulators [24-28].

The main contents of the paper mainly focus on the comparison of the controllers based on the early works and further describe the nonlinear behavior of the manipulator under controlling. In Section 2, modeling methods of the nonlinear dynamics of manipulator system are introduced. Some new synchronized control strategies are proposed, including neuron synchronization controller, improved OPCL synchronization controller, and MRACPD synchronization controller. Then, in Section 3, dynamic stability of the controlled synchronization of manipulator
TABLE 1: Structure parameter values of the 2-DOF master manipulator.

\begin{tabular}{lcccc}
\hline Link $i$ & $I_{z z}\left(\mathrm{kgm}^{2}\right)$ & $m_{i}(\mathrm{~kg})$ & $L_{c i}(\mathrm{~m})$ & $L_{i}(\mathrm{~m})$ \\
\hline 1 & 0.083 & 1 & 0.5 & 1 \\
2 & 0.33 & 1 & 1 & 2 \\
\hline
\end{tabular}

TABLE 2: Structure parameter values of the 3-DOF master manipulator.

\begin{tabular}{lcccc}
\hline Link $i$ & $I_{z z}\left(\mathrm{kgm}^{2}\right)$ & $m_{i}(\mathrm{~kg})$ & $L_{c i}(\mathrm{~m})$ & $L_{i}(\mathrm{~m})$ \\
\hline 0 & - & - & - & 0.09 \\
1 & 0.05 & 1.738 & 0.072 & 0.144 \\
2 & 0.05 & 1.738 & 0.072 & 0.144 \\
3 & 0.12 & 2.529 & 0.098 & 0.241 \\
\hline
\end{tabular}

system and the dynamic stability of robust adaptive synchronized controller are discussed where the unknown constructor parameters, loading variables, and external disturbance of the manipulators are involved. In Section 4, the influences of the control parameters and joint friction on the nonlinear behavior of the synchronized system are described, and the typical processes of the bifurcation and the loss of synchronism are also illustrated. Finally, some conclusions are given in Section 5.

\section{Dynamic Models of Manipulator System and Synchronization Control Methods}

Two planar manipulators are shown in Figure 1, and the corresponding parameters are shown in Tables 1 and 2, respectively. Based on Newton-Euler formula, their dynamic equations are derived as follows:

$$
\begin{gathered}
\mathbf{F}_{i}^{i-1}=\mathbf{F}_{i}^{i+1}+m_{i} \dot{\mathbf{v}}_{c i}-m_{i} \mathbf{g} \\
\mathbf{M}_{i}^{i-1}=\mathbf{M}_{i}^{i+1}-\mathbf{r}_{i, c i} \times \mathbf{F}_{i}^{i+1}+\mathbf{r}_{i-1, c i} \\
\times \mathbf{F}_{i}^{i-1}+\mathbf{I}_{i} \dot{\omega}_{i}+\omega_{i} \times \mathbf{I}_{i} \omega_{i},
\end{gathered}
$$

where $\mathbf{F}_{i}^{i-1}$ is the force of link $i+1$ on link $i, m_{i}$ is the mass of link $i, \mathbf{g}$ is the gravity vector, $\mathbf{M}_{i}^{i-1}$ is the torque of link $i+1$ on link $i, \mathbf{r}_{i-1, c i}$ is the vector from the coordinate origin $o_{i-1}$ adhering to joint $i$ to the center of mass $c_{i}, \mathbf{I}_{i}$ is the moment of inertia of link $i$ on mass center $c_{i}$.

Assume that the velocity of $c_{i}$ is $\mathbf{v}_{c i}$, where $c_{i}$ is the center of mass of link $i$, and the acceleration of $c_{i}$ is $\dot{\mathbf{v}}_{c i}$. The link $i$ rotates freely around $c_{i}$ with angular velocity $\omega_{i}$ and angular acceleration $\dot{\omega}_{i}$.

Define $\mathbf{q}$ as the joint variable, where $\mathbf{q}=\left\{\begin{array}{ll}\theta_{1} & \theta_{2}\end{array}\right\}^{T}$ for the 2-DOF manipulator and $\mathbf{q}=\left\{\begin{array}{lll}\theta_{1} & \theta_{2} & \theta_{3}\end{array}\right\}^{T}$ for the 3-DOF manipulator. The differential equation of motion of the manipulator is established including joint friction $\mathbf{f}(\dot{\mathbf{q}})$ and joint rigid $K$ as follows:

$$
\mathbf{M}(\mathbf{q}) \ddot{\mathbf{q}}+\mathbf{C}(\mathbf{q}, \dot{\mathbf{q}}) \dot{\mathbf{q}}+\mathbf{g}(\mathbf{q})=\tau-\mathbf{f}(\dot{\mathbf{q}})-K\left(\mathbf{q}-\mathbf{q}_{0}\right),
$$




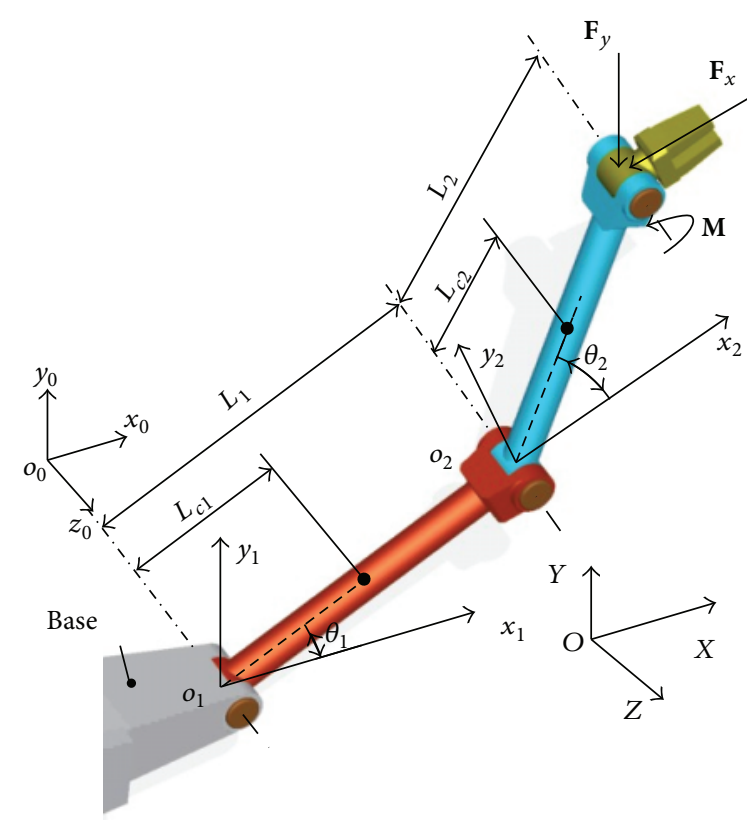

(a) A planar 2-DOF manipulator

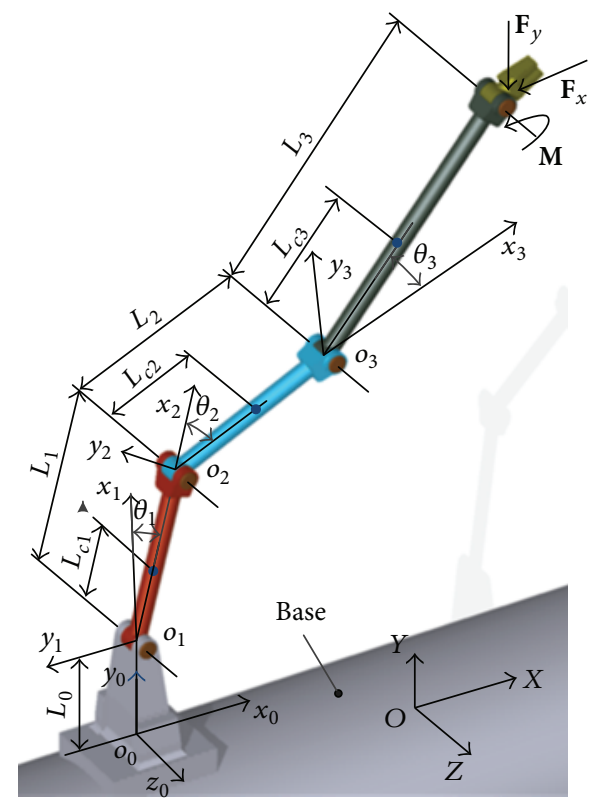

(b) A planar 3-DOF manipulator

FigURE 1: Schematic of 2 planar manipulators.

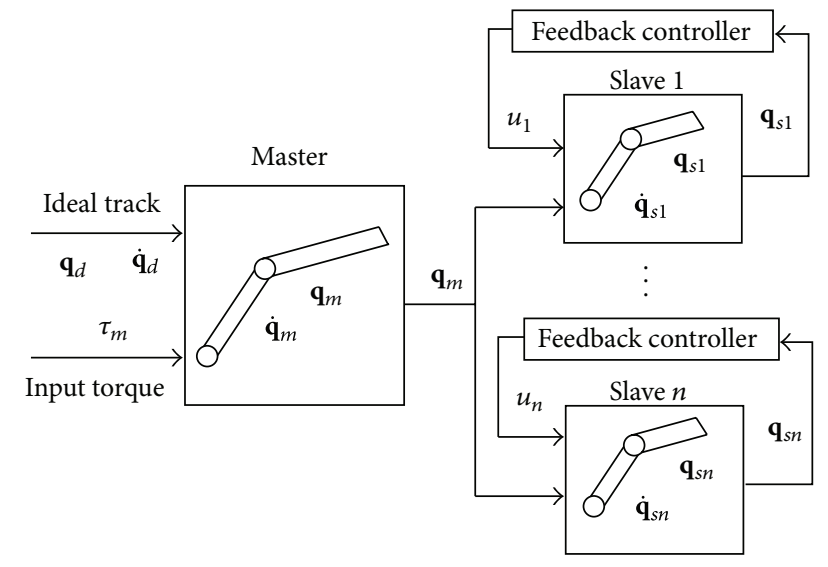

FIGURE 2: The configuration of controlled synchronization manipulators.

where the inertial force is determined by acceleration and Coriolis force, centrifugal force, and gravity load are also involved in it.

The above theory is applied in the synchronization of planar manipulators as a prototype, in which the master manipulator has the same topological mechanism with that of the slave one. The synchronization control schematic diagram for the master-slave manipulators is shown in Figure 2.

A general dynamic equation of master-slave manipulator is addressed as follows. The master system is

$$
\dot{y}=\mathbf{f}(y) \text {. }
$$

And the slave system is

$$
\dot{x}=\mathbf{g}(x)+\mathbf{u}(\mathbf{x}, \mathbf{y}),
$$

where the synchronization controller is $\mathbf{u}(\mathbf{x}, \mathbf{y})$ :

$$
\mathbf{u}(\mathbf{x}, \mathbf{y})=-\mathbf{g}(\mathbf{x})+\mathbf{f}(\mathbf{y})+\mathbf{h}(\mathbf{x}, \mathbf{y}) .
$$

The designing goal of synchronization controller is that the synchronization error of manipulators and its derivative will converge to be zero; that is, $\mathbf{e}(t) \rightarrow 0$ and $\dot{\mathbf{e}}(t) \rightarrow 0$ when $t \rightarrow \infty$. Then, the derivative of synchronization error is

$$
\dot{\mathbf{e}}=\dot{\mathbf{x}}-\dot{\mathbf{y}}=\mathbf{g}(\mathbf{x})+\mathbf{u}(\mathbf{x}, \mathbf{y})-\mathbf{f}(\mathbf{y})=\mathbf{h}(\mathbf{x}, \mathbf{y}) .
$$

According to the Lyapunov stability theory, several new type synchronization controllers can be proposed based on the feedback control strategy as introduced as follows.

2.1. Neural Synchronization Controller. For the neural synchronization controller which consisted of two reciprocal inhibition neurons, its state equations are given as follows:

$$
\begin{array}{lc}
\delta_{1} \dot{y}_{1}=c-y_{1}-b v_{1}-a x_{2}-\max (0, u) & \delta_{2} \dot{v}_{1}=x_{1}-v_{1}, \\
\delta_{1} \dot{y}_{2}=c-y_{2}-b v_{2}-a x_{1}-\max (0,-u) & \delta_{2} \dot{v}_{2}=x_{2}-v_{2}, \\
x_{1}=\max \left(0, y_{1}\right) \quad x_{2}=\max \left(0, y_{2}\right) & x_{\text {out }}=x_{1}-x_{2},
\end{array}
$$

where $\delta_{1}$ and $\delta_{2}$ are two time constants, $a$ and $b$ are the weights of mutual inhibition and self-inhibition, respectively, $c$ is the excitatory tonic input, $y_{1}$ and $y_{2}$ are the membrane potential, $v_{1}$ and $v_{2}$ are the self-inhibition, and $u$ and $x_{\text {out }}$ are the input and output of control system, respectively. 

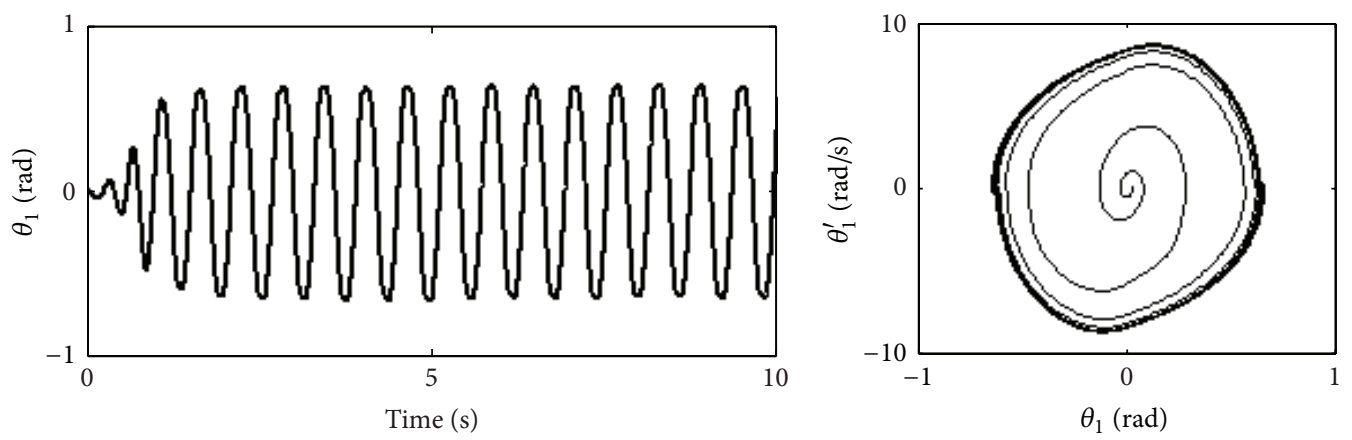

(a) Time history of and phase plane portrait of $\theta_{1}$
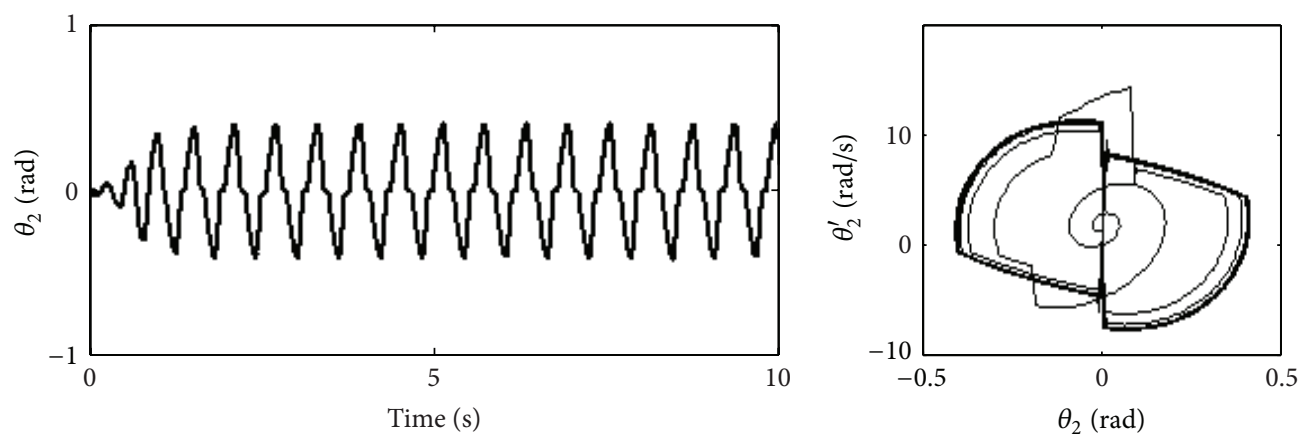

(b) Time history of and phase plane portrait of $\theta_{2}$

FIGURE 3: The motion behavior of small regular swing of a 2-link manipulator.

Apply the controller on the 2-DOF manipulator in Figure 1(a). When the two links begin to swing, the first link gets energy from the second link by using the neural synchronization controller. The whole system will come into rhythmic swing state under well-tuned controller parameter of $\theta_{2}$, which is shown in Figure 3 .

\subsection{Improved OPCL (Open-Plus-Close-Loop) Synchronization} Controller. An improved OPCL controller is designed to achieve synchronization motions based on chaos control method which consists of an amplifier and a limiter. This control method is proved to be asymptotically stable based on the Lyapunov theory given suitable control parameters. The controlled synchronizations of both the small swing and giant rotating motions of a 2-link manipulator are achieved based on the proposed improved OPCL controller. The controlled system is linearized in the neighborhood of the goal value via Taylor expansion as follows:

$$
\begin{aligned}
\ddot{\mathbf{q}}_{s}= & \mathbf{F}\left(\mathbf{q}_{s}, \dot{\mathbf{q}}_{s}, t\right)=\mathbf{F}\left(\mathbf{q}_{m}-\mathbf{e}, \dot{\mathbf{q}}_{m}-\dot{\mathbf{e}}, t\right) \\
= & \mathbf{F}\left(\mathbf{q}_{m}, \dot{\mathbf{q}}_{m}, t\right)-\left(\frac{\partial \mathbf{F}\left(\mathbf{q}_{m}, \dot{\mathbf{q}}_{m}, t\right)}{\partial \mathbf{q}_{m}}\right) \mathbf{e} \\
& -\left(\frac{\partial \mathbf{F}\left(\mathbf{q}_{m}, \dot{\mathbf{q}}_{m}, t\right)}{\partial \dot{\mathbf{q}}_{m}}\right) \dot{\mathbf{e}}+o^{2}\left(\mathbf{q}_{m}, \dot{\mathbf{q}}_{m}\right) \\
= & \mathbf{F}\left(\mathbf{q}_{m}, \dot{\mathbf{q}}_{m}, t\right)+\mathbf{J}_{\mathbf{q} m} \mathbf{e}+\mathbf{J}_{\dot{\mathbf{q}} m} \dot{\mathbf{e}}+o^{2}\left(\mathbf{q}_{m}, \dot{\mathbf{q}}_{m}\right),
\end{aligned}
$$

where $\mathbf{J}_{\mathbf{q} m}$ and $\mathbf{J}_{\dot{\mathbf{q}} m}$ are Jacobian matrices of $\mathbf{F}\left(\mathbf{q}_{m}, \dot{\mathbf{q}}_{m}, t\right)$, with respect to $\mathbf{q}_{m}, \dot{\mathbf{q}}_{m}$, respectively. The improved OPCL controller for the system is designed as

$$
\mathbf{U}=\ddot{\mathbf{q}}_{s}-\mathbf{F}\left(\mathbf{q}_{m}, \dot{\mathbf{q}}_{m}, t\right)-\mathbf{J}_{\mathbf{q} m} \mathbf{e}-\mathbf{J}_{\dot{\mathbf{q}} m} \dot{\mathbf{e}}+A \dot{\mathbf{e}}+B \mathbf{e},
$$

where the term of $\ddot{\mathbf{q}}_{s}-\mathbf{F}\left(\mathbf{q}_{m}, \dot{\mathbf{q}}_{m}, t\right)$ is the open-loop part and the term of $-\mathbf{J}_{\mathbf{q} m} \mathbf{e}-\mathbf{J}_{\mathbf{q} m} \dot{\mathbf{e}}+A \dot{\mathbf{e}}+B \mathbf{e}$ is the closed-loop part. The coefficient matrices of $A$ and $B$ are diagonal.

2.3. MARC-PD Synchronization Controller Based on PD Gains. In order to achieve the controlled synchronization (either ender motion or trajectory tracing synchronization) of manipulator system which moves in high speed together with changing loads, an improved model reference adaption control with PD gain (viz. MRAC-PD controller) is proposed to realize the desired motion, namely, synchronization.

After its global stability of the synchronization based on MRAC-PD method is proved, the effects due to the variation of control parameters have been investigated by numerical simulations.

For example, an improved MARC-PD controller is applied on a two-link manipulator to obtain the synchronization motions including small swing and giant rotation.

The principle of synchronization of ender trajectory tracing based on MRAC-PD controller is introduced as follows.

The model of controlled manipulator system is defined as

$$
\ddot{\mathbf{q}}_{s}+\mathbf{M}_{s}^{-1}\left(\mathbf{q}_{s}\right) \mathbf{C}_{s}\left(\mathbf{q}_{s}, \dot{\mathbf{q}}_{s}\right) \dot{\mathbf{q}}_{s}+\mathbf{M}_{s}^{-1}\left(\mathbf{q}_{s}\right) \mathbf{g}_{s}\left(\mathbf{q}_{s}\right)=\mathbf{M}_{s}^{-1}\left(\mathbf{q}_{s}\right) \tau_{s} \text {. }
$$




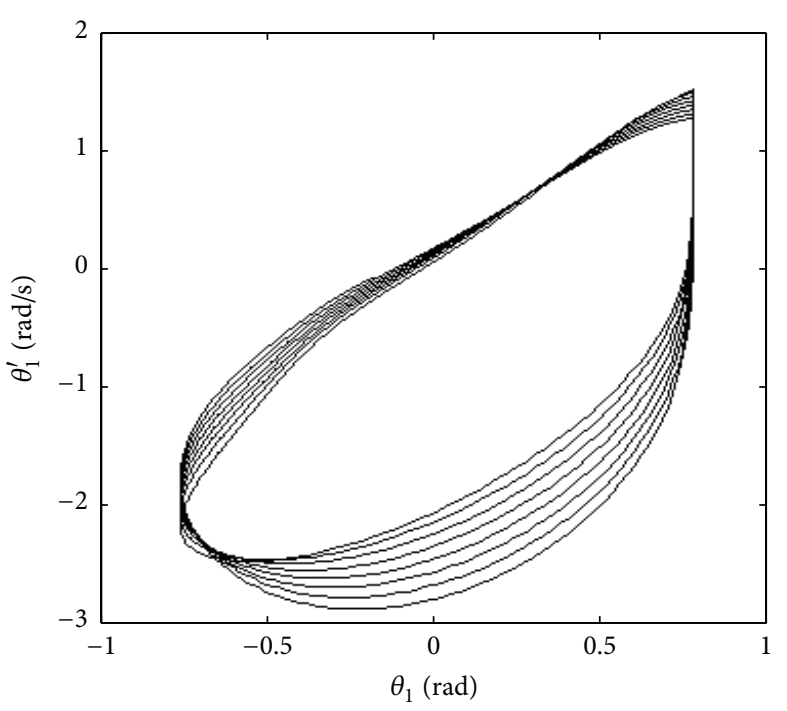

(a) Phase plane portrait of $\theta_{1}$

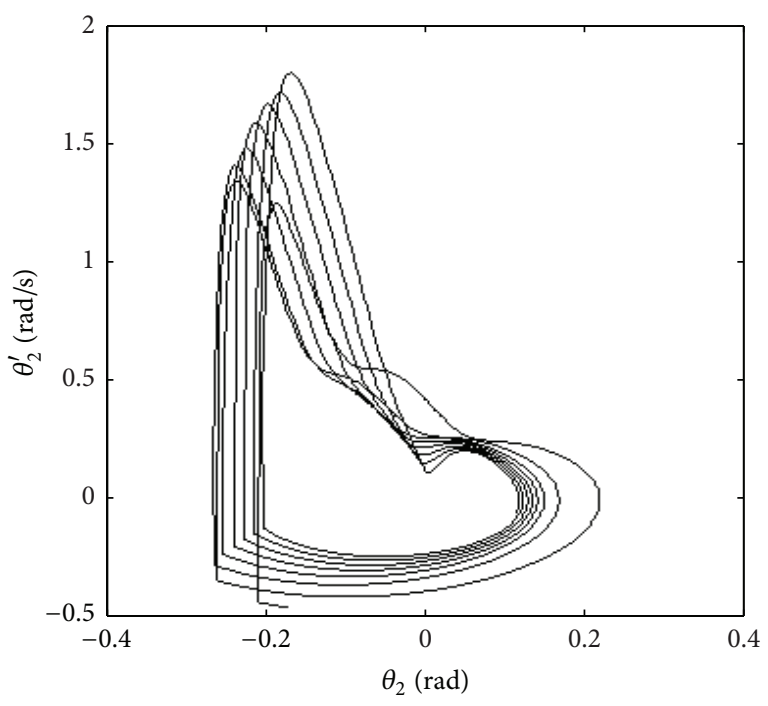

(b) Phase plane portrait of $\theta_{2}$

FIGURE 4: The synchronous motion patterns of small swing of 2-link manipulator using MRAC-PD.

The synchronous error is defined as

$$
\mathbf{e}=\mathbf{q}_{m}-\mathbf{q}_{s}
$$

and the dynamics equation of it is deduced as

$$
\begin{aligned}
\ddot{\mathbf{e}}+2 \varsigma \omega_{n} \dot{\mathbf{e}}+\omega_{n} \mathbf{e}= & \omega_{n}^{2} \mathbf{q}_{m}-\mathbf{M}_{s}^{-1}\left(\mathbf{q}_{s}\right) \boldsymbol{\tau}_{s} \\
& +\left(\mathbf{M}_{s}^{-1}\left(\mathbf{q}_{s}\right) \mathbf{C}_{s}\left(\mathbf{q}_{s}, \dot{\mathbf{q}}_{s}\right)-2 \varsigma \omega_{n}\right) \dot{\mathbf{q}}_{s} \\
& +\mathbf{M}_{s}^{-1}\left(\mathbf{q}_{s}\right) \mathbf{g}_{s}\left(\mathbf{q}_{s}\right)-\omega_{n}^{2} \mathbf{q}_{s} .
\end{aligned}
$$

Assuming the state vector of errors $\boldsymbol{\varepsilon}=[\mathbf{e}, \dot{\mathbf{e}}]^{T}$, the differential equation of it can be deduced as

$$
\dot{\boldsymbol{\varepsilon}}=\mathbf{A} \boldsymbol{\varepsilon}-\left[\begin{array}{c}
0 \\
\mathbf{M}_{s}^{-1}\left(\mathbf{q}_{s}\right)
\end{array}\right] \boldsymbol{\tau}_{s}+\left[\begin{array}{c}
0 \\
\Delta
\end{array}\right],
$$

where $\Delta$ is the model error.

It is known that, if the real parts of the eigenvalue of the coefficient matrix $\mathbf{A}$ are negative, the synchronous error shown in (12) is proved to be zero in asymptotic stability.

The control item $\widehat{e}$ according to the PD gain is defined as

$$
\widehat{e}=p_{1} e+p_{2} \dot{e}
$$

Taking the stability of Lyapunov function of the controlled system into account, the MRAC-PD controller is defined as follows:

$$
\tau_{s}=k_{0} q_{m}(t)+k_{1} q_{s}(t)+k_{2} \dot{q}_{s}(t),
$$

where the coefficients are defined as follows: $\dot{k}_{0}=\lambda_{0} \hat{e} q_{m}(t)$, $\dot{k}_{1}=\lambda_{1} \hat{e} q_{s}(t)$, and $\dot{k}_{2}=\lambda_{2} \hat{e} q_{s}(t)$.

Apply the controller on the 2-DOF manipulator in Figure 1(a). The results are shown in Figure 4, where the phase space trajectories of $q_{s 1}, q_{s 2}$ indicate the obtained unchangeable patterns during the motion synchronizations of small swing.

\section{Dynamics Stability of Controlled Synchronization of Manipulator under Complicated Conditions}

In order to eliminate the effectiveness by uncertainty structure parameters, friction compensation, unknown load, and flexible joint, the on-time estimation method of structure parameters and state variables of manipulator is built to achieve the synchronization of manipulator system. That is, the stability of controlled synchronization of manipulator for the casings of disturbance, uncertainty parameter, and unknown structure is improved as enhancing the robust function of synchronization controller.

3.1. Dynamics of Controlled Synchronization of Manipulator System Based on Estimation of Structure Parameters. Under the condition of structure parameters of the slave manipulator in a master-slave system being unknown, the exact expression of $\mathbf{g}(\mathbf{x})$ should be estimated in the process of synchronization control. In this case, the synchronization controller $\mathbf{u}(\mathbf{x}, \mathbf{y})$ is defined as the following three terms:

$$
\mathbf{u}(\mathbf{x}, \mathbf{y})=-\widehat{\mathbf{g}}(\mathbf{x})+\mathbf{f}(\mathbf{y})+\mathbf{h}(\mathbf{x}, \mathbf{y})
$$

where $\widehat{\mathbf{g}}(\mathbf{x})$ is the estimator of $\mathbf{g}(x), \mathbf{f}(\mathbf{y})$ is the master function, and $\mathbf{h}(\mathbf{x}, \mathbf{y})$ is a special control term.

In order to obtain $\widehat{\mathbf{g}}(\mathbf{x})$, some assumptions are used and $\widehat{\mathbf{g}}(\mathbf{x})$ is expressed linearly as follows:

$$
\widehat{\mathbf{g}}(\mathbf{x})=\widehat{\mathbf{M}}(\mathbf{x})+\mathbf{X}(x) \widehat{\mathbf{N}}+\mathbf{C}_{\text {const }} \text {, }
$$

where $\widehat{\mathbf{M}}(\mathbf{x})$ is the estimation of nonlinear item, $\widehat{\mathbf{N}}$ is the estimator of the linear term containing unknown structure parameters, $\mathbf{X}(x)$ is the linear term containing state variables, and $\mathbf{C}$ is a constant. 
Then, the synchronization error is rewritten as follows:

$$
\begin{aligned}
\widetilde{\mathbf{g}}(\mathbf{x}) & =\widehat{\mathbf{g}}(\mathbf{x})-\mathbf{g}(\mathbf{x}) \\
& =(\widehat{\mathbf{M}}(\mathbf{x})-\mathbf{M}(\mathbf{x}))+\mathbf{X}(x)(\widehat{\mathbf{N}}(\mathbf{x})-\mathbf{N}(\mathbf{x})) \\
& =\widetilde{\mathbf{M}}(\mathbf{x})+\mathbf{X}(\mathbf{x}) \widetilde{\mathbf{N}}(\mathbf{x}),
\end{aligned}
$$

where $\widetilde{\mathbf{g}}(\mathbf{x})$ is the estimation error of $\mathbf{g}(\mathbf{x}), \widetilde{\mathbf{M}}(\mathbf{x})$ is the estimation error of nonlinear item, and $\widetilde{\mathbf{N}}(\mathbf{x})$ is the estimation error of linear item affected by the system parameter.

In particular, when nonlinear item $\mathbf{M}(\mathbf{x})=0$, then

$$
\widetilde{\mathbf{g}}(\mathbf{x})=\mathbf{X}(\mathbf{x}) \widetilde{\mathbf{N}}(\mathbf{x}) .
$$

So the synchronization error equation is defined as

$$
\begin{aligned}
\dot{\mathbf{e}}=\dot{\mathbf{x}}-\dot{\mathbf{y}} & =\mathbf{g}(\mathbf{x})+\mathbf{u}(\mathbf{x}, \mathbf{y})-\mathbf{f}(\mathbf{y}) \\
& =\mathbf{g}(\mathbf{x})-\widehat{\mathbf{g}}(\mathbf{x})+\mathbf{h}(\mathbf{x}, \mathbf{y}) \\
& =-\widetilde{\mathbf{g}}(\mathbf{x})+\mathbf{h}(\mathbf{x}, \mathbf{y})=-\mathbf{X}(\mathbf{x}) \widetilde{\mathbf{N}}(\mathbf{x})+\mathbf{h}(\mathbf{x}, \mathbf{y}) .
\end{aligned}
$$

Considering the contribution of the estimation error $\widetilde{\mathbf{g}}(\mathbf{x})$, the adaptive law according to the Lyapunov stability theory is defined as follows:

$$
\frac{d \widehat{\mathbf{N}}}{d t}=-\mathbf{X}^{T} \mathbf{e}
$$

If needed, some reasonable synchronization controllers can also be designed to realize the stable controlled synchronization under the situations of both the linear and the nonlinear coupling parameters being unknown.

The above theory is applied in the synchronization of two 3-DOF planar manipulators in Figure 1(b). Each joint of the slave will trace the corresponding joint trajectory of the master in a synchronization way. The synchronization control schematic diagram for the master-slave manipulators is shown as follows.

According to the characteristics of (2), the dynamic equation can be linearized and the adaptive method can guarantee the synchronization stability of the system with unknown slave parameters. Then, (2) can be rewritten as

$$
\mathbf{M}_{s}\left(\mathbf{q}_{s}\right) \ddot{\mathbf{q}}_{m}+\mathbf{C}_{s}\left(\mathbf{q}_{s}, \dot{\mathbf{q}}_{s}\right) \dot{\mathbf{q}}_{m}+\mathbf{g}_{s}\left(\mathbf{q}_{s}\right)=\Phi\left(\mathbf{q}_{s}, \dot{\mathbf{q}}_{s}, \dot{\mathbf{q}}_{m}, \ddot{\mathbf{q}}_{m}\right) \mathbf{P},
$$

where $\mathbf{P} \in R_{17 \times 1}, \boldsymbol{\Phi}\left(\mathbf{q}_{s}, \dot{\mathbf{q}}_{s}, \dot{\mathbf{q}}_{m}, \ddot{\mathbf{q}}_{m}\right) \in R_{3 \times 17} . \mathbf{P}$ is a vector which contains all the constant parameters besides the angular information at each joint, and $\Phi\left(\mathbf{q}_{s}, \dot{\mathbf{q}}_{s}, \dot{\mathbf{q}}_{m}, \ddot{\mathbf{q}}_{m}\right)$ does not contain any inertial characteristics and constant parameters of the manipulator.

Let $\dot{\mathbf{q}}_{r}=\dot{\mathbf{q}}_{m}-r \mathbf{e}$ and $\dot{\mathbf{e}}_{r}=\dot{\mathbf{e}}+r \mathbf{e}(r>0)$, and at last the novel controller for synchronization of the master-slave manipulators corresponding to (19) and (21) can be written as follows:

$$
\begin{gathered}
\tau_{s}=\boldsymbol{\Phi}\left(\mathbf{q}_{s}, \dot{\mathbf{q}}_{s}, \dot{\mathbf{q}}_{r}, \ddot{\mathbf{q}}_{r}\right) \widehat{\mathbf{P}}-\mathbf{K}_{p} \mathbf{e}-\mathbf{K}_{v} \dot{\mathbf{e}} \\
\dot{\widehat{\mathbf{P}}}=-\mathbf{A} \boldsymbol{\Phi}\left(\mathbf{q}_{s}, \dot{\mathbf{q}}_{s}, \dot{\mathbf{q}}_{r}, \ddot{\mathbf{q}}_{r}\right)^{T} \dot{\mathbf{e}}_{r},
\end{gathered}
$$

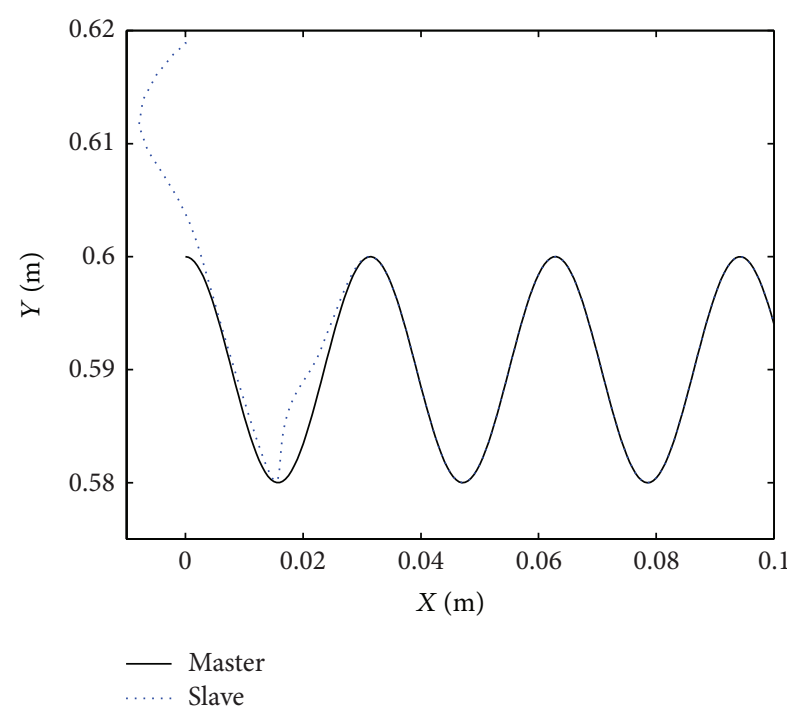

FIGURE 5: Ender trajectories of the master-slave manipulators.

where $\widehat{\mathbf{P}}$ is the estimation of the parameter vector $\mathbf{P}$, and it is continuously adjusting according to the synchronization error. Through the adaptive law in (24), the adaptive controller can effectively control the manipulator.

The simulation results of the synchronization of two 3DOF planar manipulators based on the proposed control method are plotted in Figure 5.

3.2. Dynamics Stability of Manipulator Based on Robust Self-Adaptive Synchronization Control. Considering the unknown model error and possible disturbance, the dynamic equation of a manipulator is defined as

$$
\boldsymbol{\tau}=\mathbf{M}(\mathbf{q}) \ddot{\mathbf{q}}+\mathbf{C}(\mathbf{q}, \dot{\mathbf{q}}) \dot{\mathbf{q}}+\mathbf{g}(\mathbf{q})+\Delta(\mathbf{q}, \dot{\mathbf{q}}),
$$

where $\Delta(\mathbf{q}, \dot{\mathbf{q}})$ is the model error. A robust controller of synchronous trajectory tracing is designed to guarantee the tracing error which is defined as $e(t)=q(t)-q_{d}(t)$ to be asymptotically limited when the model error is limited or to be asymptotically zero if the model error is zero.

Given an auxiliary signal, $s=\dot{e}+\delta e$, and that $\delta>0$ is a stability constant, the error equation of the controlled synchronization system is derived as follows:

$$
\mathbf{M}(\mathbf{q}) \dot{\mathbf{s}}=-\mathbf{C}(\mathbf{q}, \dot{\mathbf{q}}) s+\phi-\Delta(\mathbf{q}, \dot{\mathbf{q}})+\mathbf{u} .
$$

The robust condition of a synchronization controller is that the positive definite function $\rho(e, \dot{e})$ must be defined for any $\Delta(q, \dot{q})$ to meet the following inequality constraint:

$$
\|\Delta(q, \dot{q})\| \leq \rho(e, \dot{e}) .
$$

The proposed synchronization controller consists of two parts: one is the feed forward controller and the other is the feedback controller; they are as follows:

$$
u_{1}=-\mathbf{K} s-\phi, \quad u_{2}=-v,
$$

where $\mathbf{K}$ is a positive feed forward coefficient, $\phi=\mathbf{M}(q) \delta \dot{e}+$ $\mathbf{C}(q, \dot{q}) \delta e$, and $v=s \rho^{2}(e, \dot{e}) /(\|s\| \rho(e, \dot{e})+\varepsilon)$ in which $\varepsilon>0$ is a definite constant. 


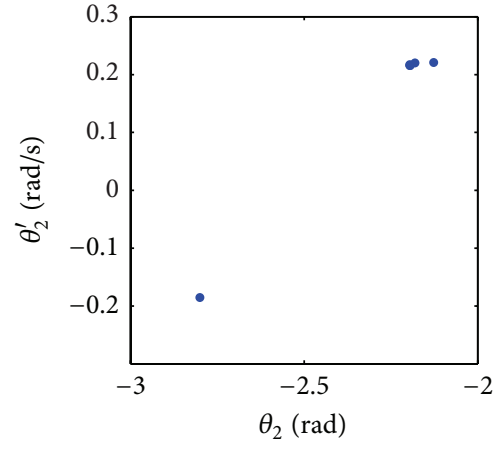

(a) Poincare map of multiple periodic motion

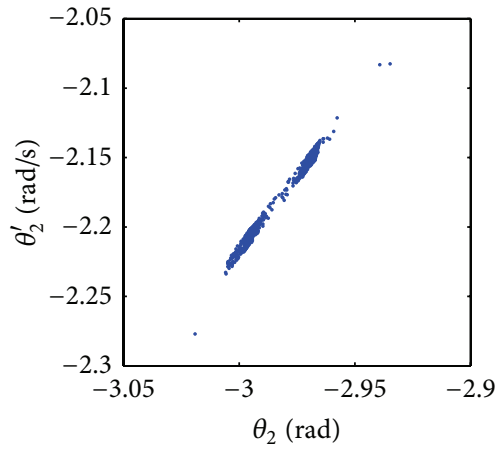

(b) Poincare map of quasiperiodic motion

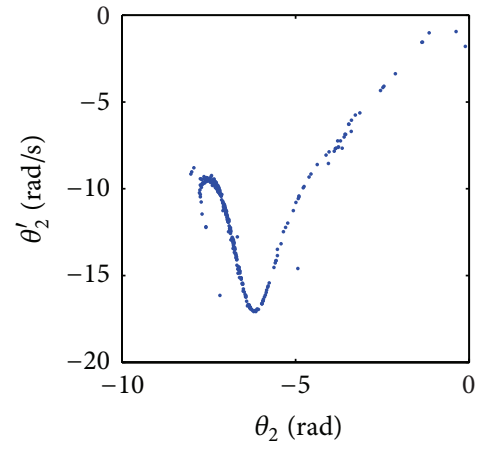

(c) Poincare map of chaotic motions

FIGURE 6: Poincare maps of the joint motions of 2-link manipulator under different OPCL control parameters.

\section{Nonlinear Dynamic Behavior of the Controlled Synchronous Manipulator System}

From the viewpoint of the theories of nonlinear dynamics, bifurcations, and chaos, bifurcations and possible chaos could appear in the controlled synchronization processes of the manipulator system. The possible motion patterns of it include single periodic, multiple periodic, quasiperiodic, and chaotic. While the motion patterns and also the dynamic characteristics of manipulator under different synchronization controls will be greatly affected by the unavoidable joint frictions and especially designed control parameters.

4.1. Complicated Motions of a 2-Link Manipulator under OPCL Synchronization Control. Changing of control parameters can affect the synchronization motions such as small swing and giant rotating of a 2-link manipulator under OPCL control greatly and induce different motions of single periodic, multiple periodic, quasiperiodic, and chaotic ones. The transition processes of the two kinds of synchronization motions are also determined by the OPCL parameters.

Assume that the structure parameter values of the 2-link manipulator in Figure 1(a) are unchangeable, and the two joint angular trajectories are expected as harmonic ones; the obtained Poincare map of the joint angles is shown in Figure 6 when the feed forward coefficients of $\mathbf{A}$ and $\mathbf{B}$ change. For the chaotic case, the calculated Lyapunov exponents of the two joint angles are positive; that is, the biggest Lyapunov exponents of them are 0.5496 and 0.1431 , respectively.

\subsection{Nonlinear Behavior of a Controlled Synchronous Manip-} ulator considering Joint Friction. The unavoidable joint frictions will greatly affect the synchronization of the 2-link manipulator under OPCL control. Based on the Stribeck force model of joint friction, the influences of the viscous friction coefficient, static friction force, and Coulomb friction force on the synchronization motions of the 2-link manipulator under OPCL control are compared. The possible motions of it can be single periodic, multiple periodic, quasiperiodic, and chaotic if the joint viscous frictions are changed. Just

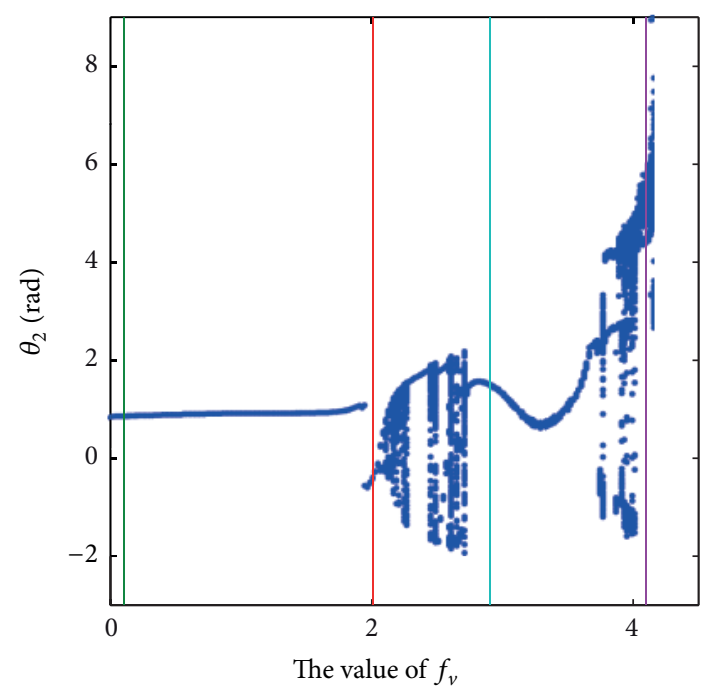

FIGURE 7: Bifurcations of joint angular motion along viscous frictions.

as shown in Figure 7, the joint angular motion bifurcation happens along the viscous friction changing from 0 to 4.15 .

When the value of viscous friction is constant, the static friction and Coulomb friction will also affect the motions of manipulator too. Letting viscous friction $f_{v}=4.1$, different static friction coefficients of joint angular lead to quasiperiodic and chaotic motions of the manipulator, respectively, as shown in Figure 8.

\section{Conclusions}

The nonlinear dynamics of the manipulator system which is controlled to achieve synchronization motions is investigated in the paper. Firstly, the modeling approach of manipulator together with the corresponding synchronization control strategies is stated in detail. The dynamic phenomena of swing motions of a two-link manipulator controlled by a neural controller have been described thoroughly. The motion characteristics of two kinds of synchronization motions are simulated. An improved OPCL control method is proposed 


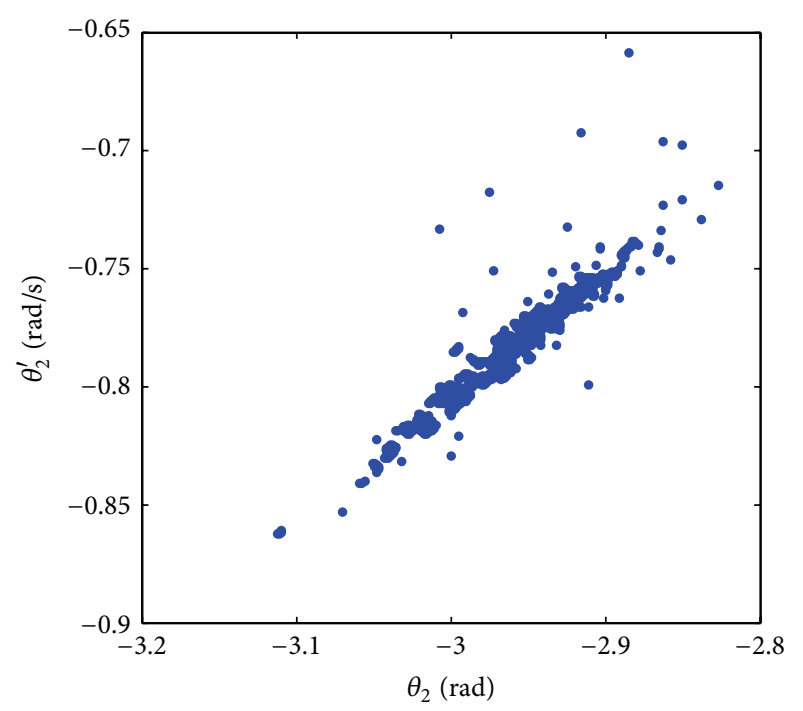

(a) Quasiperiodic motion

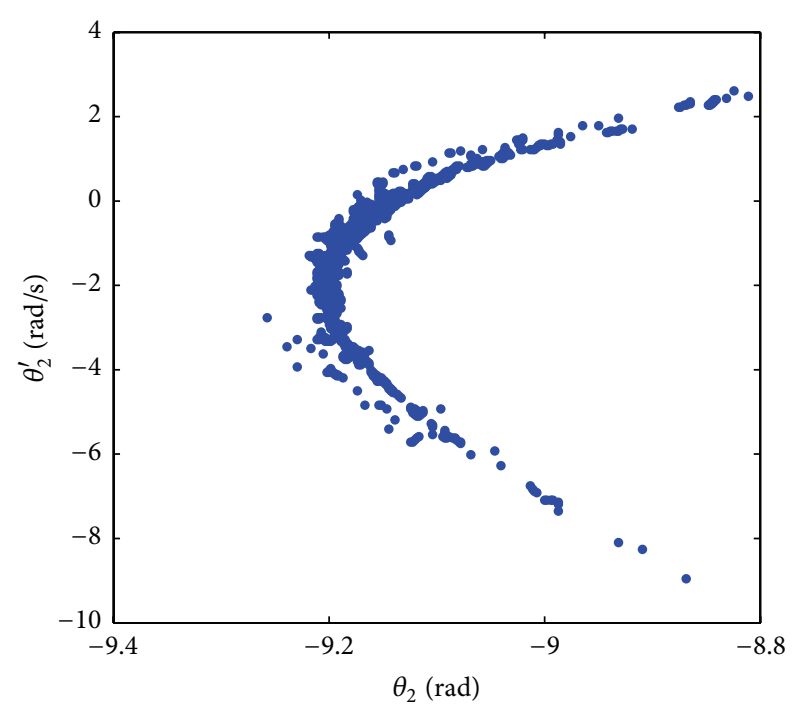

(b) Chaotic motion

FIGURE 8: Joint angular motions with different viscous frictions.

to achieve synchronization motions of both small swing and giant rotating for a two-link manipulator too. MRACPD synchronization controller is proposed to achieve more accurate trajectory tracing and synchronous motions for 3-DOF manipulator system under the conditions of high operating speed and unknown structure parameters.

The dynamic stability of controlled synchronization of manipulator system is also explained. An estimation based synchronization control method of manipulator system is investigated to eliminate the influences of unknown structure parameters, joint friction, and unknown load. The new synchronization method can improve the robustness of the manipulator system. The controlled synchronization stability is also improved even in case of disturbance, uncertainty parameter, and unknown structure.

Some complicated nonlinear behavior of the controlled synchronizations of manipulator is investigated including multiperiodic motions and bifurcation. Along the changing of the control parameters, viscous friction and static friction, the synchronous manipulator can present single-periodic, multiperiodic, quasiperiodic, and chaotic motions.

\section{Conflict of Interests}

The authors declare that there is no conflict of interests regarding the publication of this paper.

\section{Acknowledgment}

The authors gratefully acknowledge that the work was supported by the Open Project of State Key Laboratory of Robotics of Chinese Academy of Science (Grant no. 2012O04).

\section{References}

[1] A. Pikovsky, M. Rosenblum, and J. Kurths, Synchronization, A Universal Concept in Nonlinear Sciences, Cambridge University Press, 2001.

[2] I. I. Blekhman, Synchronization in Science and Technology, ASME Press, New York, NY, USA, 1988.

[3] I. I. Blekhman, P. S. Landa, and M. G. Rosenblum, "Synchronization and chaotization in interacting dynamical systems," ASME Applied Mechanical Review, vol. 48, pp. 733-752, 1995.

[4] M. Rosenblum and A. Pikovsky, "Synchronization: from pendulum clocks to chaotic lasers and chemical oscillators," Contemporary Physics, vol. 44, no. 5, pp. 401-416, 2003.

[5] W. Lindsey, Synchronization Systems in Communication and Control, Prentice-Hall, Upper Saddle River, NJ, USA, 1972.

[6] G. Chen and X. Dong, From Chaos to Order: Methodologies, Perspectives and Applications, World Scientific, Singapore, 1998.

[7] B. Wen, J. Fan, C. Zhao, and W. Xiong, Vibratory and Controlled Synchronization Engineering, Science Press of China, 2012.

[8] I. I. Blekhman, A. L. Fradkov, O. P. Tomchina, and D. E. Bogdanov, "Self-synchronization and controlled synchronization: general definition and example design," Mathematics and Computers in Simulation, vol. 58, no. 4-6, pp. 367-384, 2002.

[9] Y. Koren, "Cross-coupled biaxial computer control for manufacturing systems," Journal of Dynamic Systems, Measurement and Control, Transactions of the ASME, vol. 102, no. 4, pp. 265272, 1980.

[10] A. Rodriguez-Angeles and H. Nijmeijer, "Mutual synchronization of robots via estimated state feedback: a cooperative approach," IEEE Transactions on Control Systems Technology, vol. 12, no. 4, pp. 542-554, 2004.

[11] H. Nijmeijer, "A dynamical control view on synchronization," Physica D, vol. 154, no. 3-4, pp. 219-228, 2001.

[12] H. Nijmeijer and A. Rodriguez-Angeles, Synchronization of Mechanical Systems, World Scientific, 2003.

[13] M. Brunt, Coordination of Redundant Systems, Technical University Delft, Dordrecht, The Netherlands, 1998. 
[14] L. Feng, Y. Koren, and J. Borenstein, "Cross-coupling motion controller for mobile robots," IEEE Control Systems Magazine, vol. 13, no. 6, pp. 35-43, 1993.

[15] M. Tomizuka, J.-S. Hu, T.-C. Chiu, and T. Kamano, "Synchronization of two motion control axes under adaptive feedforward control," Journal of Dynamic Systems, Measurement and Control, Transactions of the ASME, vol. 114, no. 2, pp. 196-203, 1992.

[16] L. Ren, J. K. Mills, and D. Sun, "Adaptive synchronized control for a planar parallel manipulator: theory and experiments," Journal of Dynamic Systems, Measurement and Control, Transactions of the ASME, vol. 128, no. 4, pp. 976-979, 2006.

[17] D. Sun and J. K. Mills, "Adaptive synchronized control for coordination of multirobot assembly tasks," IEEE Transactions on Robotics and Automation, vol. 18, no. 4, pp. 498-510, 2002.

[18] D. Sun, R. Lu, J. K. Mills, and C. Wang, "Synchronous tracking control of parallel manipulators using cross-coupling approach," International Journal of Robotics Research, vol. 25, no. 11, pp. 1137-1147, 2006.

[19] D. Sun, X. Shao, and G. Feng, "A model-free cross-coupled control for position synchronization of multi-axis motions: theory and experiments," IEEE Transactions on Control Systems Technology, vol. 15, no. 2, pp. 306-314, 2007.

[20] B. L. Walcott and S. H. Zak, "State observation of nonlinear uncertain dynamical systems," IEEE Transactions on Automatic Control, vol. 32, no. 2, pp. 166-170, 1987.

[21] H. Bolandi and A. F. Ehyaei, "Trajectory planning of two cooperative mobile manipulators under closed-chain and differential constraints," International Journal of Innovative Computing, Information and Control, vol. 8, no. 2, pp. 1077-1102, 2012.

[22] F. Salas, M. Llama, and V. Santibanez, "A stable self-organizing fuzzy PD control for robot manipulators," International Journal of Innovative Computing, Information and Control, vol. 9, no. 5, pp. 2065-2086, 2013.

[23] R. Yang, P. Shi, and G.-P. Liu, "Filtering for discrete-time networked nonlinear systems with mixed random delays and packet dropouts," IEEE Transactions on Automatic Control, vol. 56, no. 11, pp. 2655-2660, 2011.

[24] Q. Han, "Controlled synchronization for masterslave manipulators based on observed ender trajectory," International Journal of Structural Stability and Dynamics, vol. 11, no. 6, pp. 1089-1102, 2011.

[25] Q. Han, X. Zhao, X. Li, and B. Wen, "Bifurcations of a controlled two-bar linkage motion with considering viscous frictions," Shock and Vibration, vol. 18, no. 1-2, pp. 365-375, 2011.

[26] Q. Han, X. Zhao, X. Yang, and B. Wen, "Periodic and chaotic motions of a two-bar linkage with OPCL controller," Mathematical Problems in Engineering, vol. 2010, Article ID 986319, 15 pages, 2010.

[27] Q. Han, X. Sun, X. Yang, and B. Wen, "External synchronization of two dynamical systems with uncertain parameters," Science China Technological Sciences, vol. 53, no. 3, pp. 731-740, 2010.

[28] Q. Han, X. Sun, X. Yang, and B. Wen, "External synchronization of a hysteretic system with a duffing system by feedback control strategy," International Journal of Structural Stability and Dynamics, vol. 9, no. 3, pp. 461-471, 2009. 


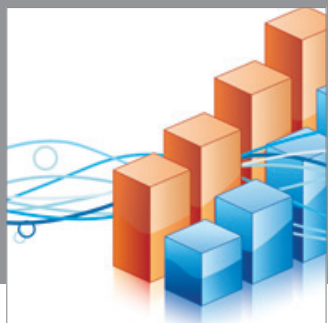

Advances in

Operations Research

mansans

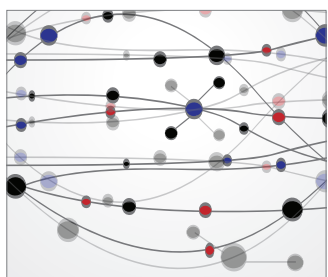

The Scientific World Journal
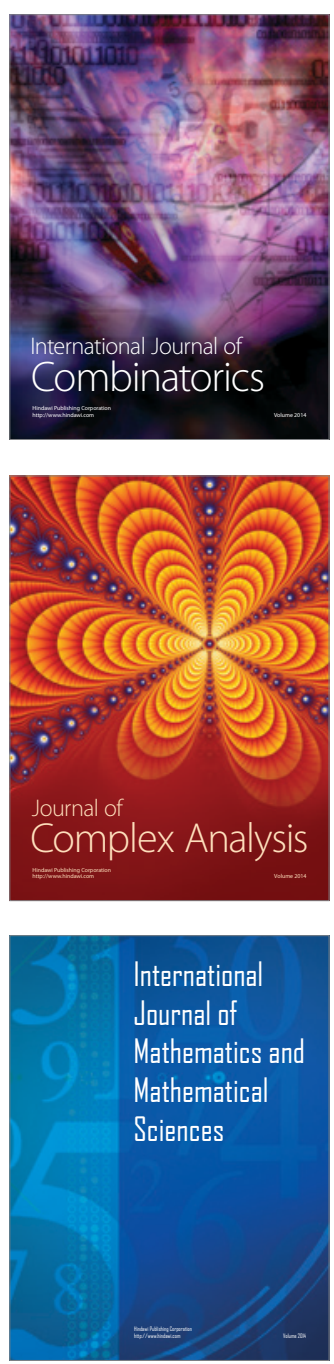
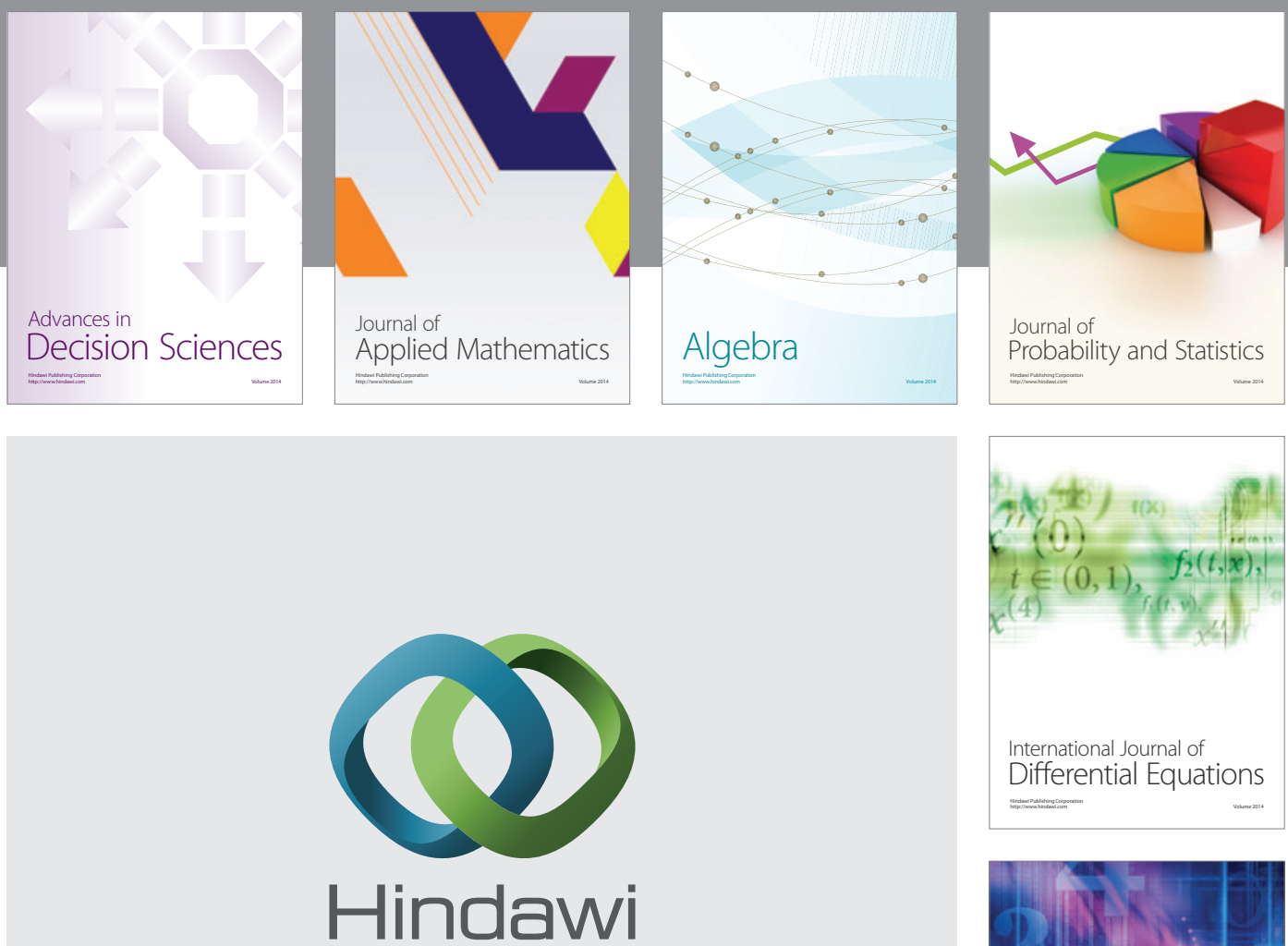

Submit your manuscripts at http://www.hindawi.com
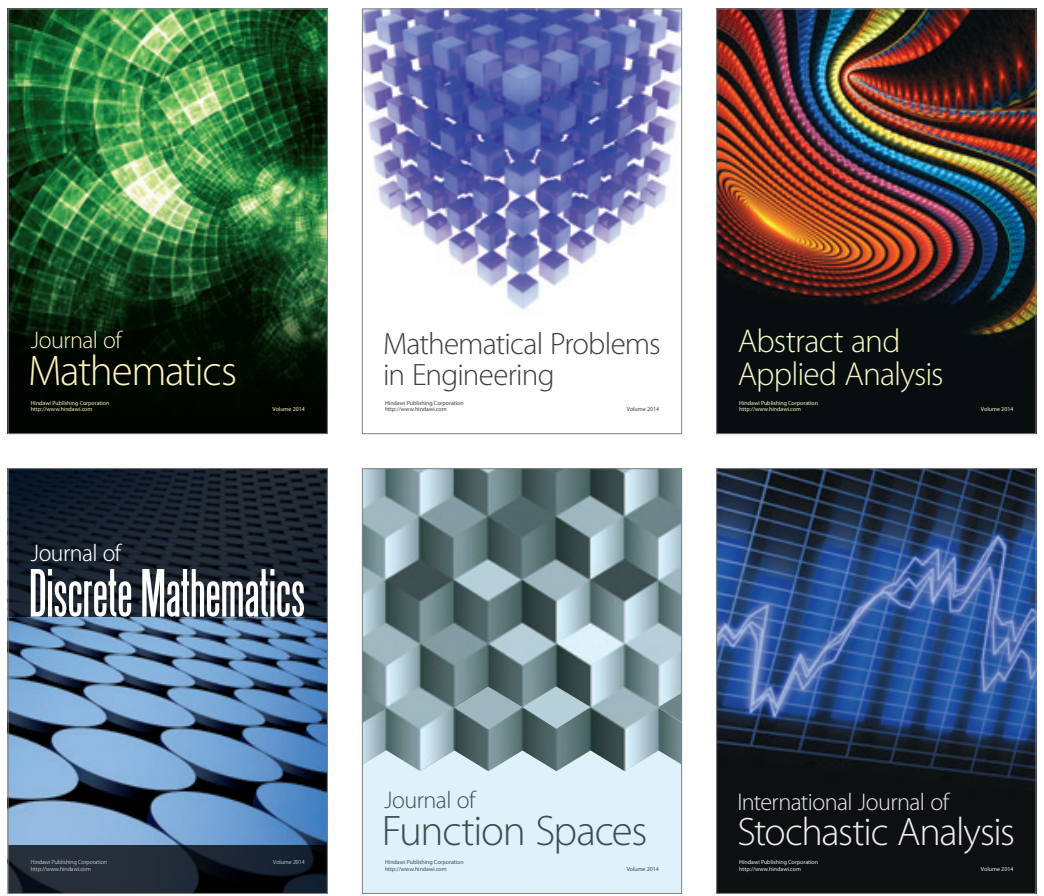

Journal of

Function Spaces

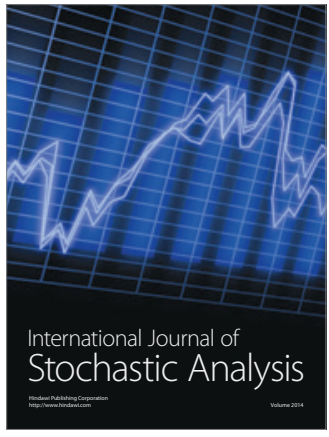

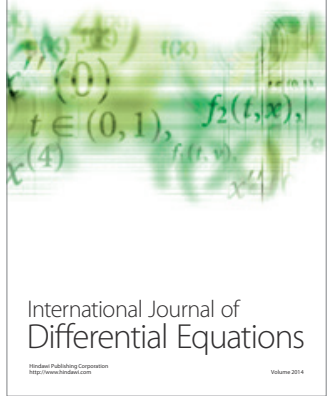
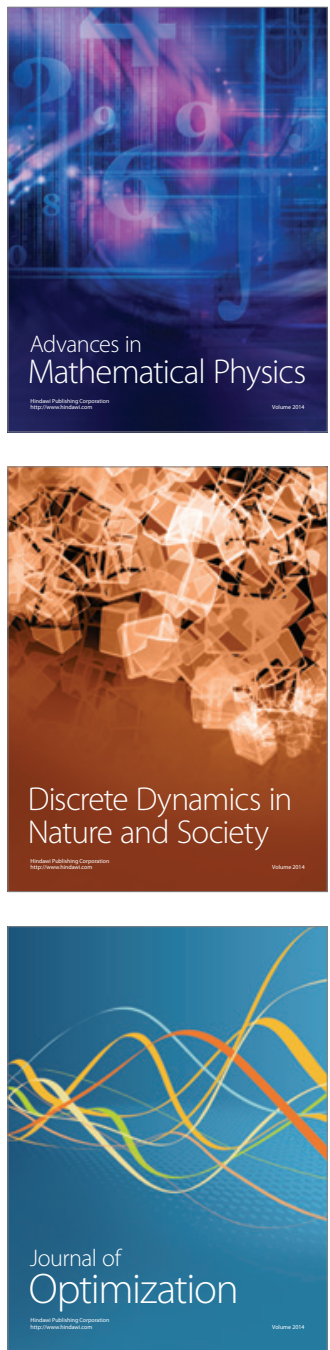